\title{
An effective medium ratio obeying meta-atom for multiband applications
}

\author{
M. J. HOSSAIN ${ }^{1 *}$, M. R. I. FARUQUE ${ }^{1}$, M. T. ISLAM ${ }^{2}$, and S. S. ISLAM ${ }^{1}$ \\ ${ }^{1}$ Space Science Center (ANGKASA), Universiti Kebangsaan Malaysia, 43600 UKM, Bangi, Selangor, Malaysia \\ ${ }^{2}$ Department of Electrical, Electronic and Systems Engineering, Universiti Kebangsan Malaysia, 43600 UKM, Bangi, Selangor, Malaysia
}

\begin{abstract}
In this paper, an effective medium ratio obeying, negative refractive index, compact meta-atom was designed, manufactured and tested for operation in a multiband microwave regime. The proposed meta-atom structure obeyed better effective medium ratio. The results of the measurement were verified by the commercially available Computer Simulation Technology (CST) Studio Suite 2014 simulator. The effective medium ratio obeying meta-atom displayed multiband response, in conjunction with negative refractive index property over a certain frequency band in the microwave frequency span. Furthermore, a few parametric analyses were completed with the meta-atom, and the effective medium parameters were investigated. The sizes and complex scattering constitutive parameters of the proposed negative refractive index, compact meta-atom are applicable for multiband applications in microwave regime.
\end{abstract}

Key words: compact, effective medium ratio, meta-atom, multiband.

\section{Introduction}

The artificially engineered split-ring resonator (SRR) meta-atom is a popular metamaterial structure, usually utilized in microwave regime. The SRR meta-atom is fabricated on a dielectric substrate, using a well-conducting agent, like copper or gold, as a form of conducting elements. The array of the SRR meta-atom can offer highly positive and/or negative value of the effective permeability over a certain frequency span [1]. In 1968, V. G. Veselgo proposed a theoretical double-negative metamaterial which was based on these negative-valued permeabilities, particularly [2]. An electromagnetic metamaterial is a structural material developed by a periodic arrangement of engineered structures called meta-atoms. An electromagnetic metamaterial can show negative permittivity and/or permeability in the effective medium region [3]. Compactness of the metamaterial depends on the effective medium ratio (EMR), which is described as a ratio between the wavelengths and unit-cell size. In the field of metamaterial research, improving the effective medium ratio is an important issue, as it governs the compactness of the metamaterial. In 2010, Abbott et al. investigated a compact capacitive loading design to improve the effective medium ratio [4]. Different types of unit-cell structures, such as split ring resonators [2], an electric-inductor-capacitor resonator [5], and a metallic short wire [6] were depicted in the microwave frequency span in an attempt to achieve metamaterial-like characteristics. On the other hand, most of the traditional unitcell structures introduce a single-band response. Due to specific applications, different types of alphabetic structures of metamaterials were proposed. A z-shaped metamaterial, proposed by Abdallah Dhouibi et al. in [7], was found to work at a single

*e-mail: jakir@siswa.ukm.edu.my band, only with a single negative property. A diamond-shaped, single-sided, left-handed metamaterial was proposed to show increased reception of power and a high transmission peak [8]. In addition, the operating band of the diamond-shaped unit-cell was X-band only, and the EMR of the unit-cell was too small. A novel hexagon meta-atom cluster was proposed as a compact metamaterial [9], whereas the metamaterial covered the S-band only, and the resonance number was 1, with EMR 2.65. In [10], a novel, two-rectangular, U-shaped, double-negative metamaterial was suggested for single-band operation. Moreover, the design of the unit-cell of the metamaterial was intended for C-band, and the EMR was of less than standard value. Theodosius et al. [11], depicted a double-negative (DNG) metamaterial of compact size with 6.9 EMR, but they utilized two different resonators at two opposing sides of the substrate. New double-negative and single-negative metamaterial analyses were performed for tri-band operation by Islam et al. [12], yet the metamaterial design was very large in size and the EMR was too small. A Greek-key pattern was then proposed as a miniaturized metamaterial [13], whereas the metamaterial covered the S-band only, with the number of resonances being 2 . A new dual-band metamaterial unit-cell construction with EMR 7.14 was proposed in [14], but it suggested single-negative property for its dual-band operation. A compact, negative-index metamaterial was analyzed for multiband operation in [15], whereas the metamaterial covered the S-band and C-band with two resonances only. Cumali et al. in [16] depicted a resonator-based metamaterial using microstrip technology with 4.76 EMR, but the metamaterial covered L-band only and the number of resonances was one.

In this study, a new, compact, double-C-shaped metamaterial unit-cell for microwave multiband applications with better EMR properties is being introduced. The proposed metamaterial demonstrates negative permittivity and negative perme- 
ability at S-band and C-band, and double negative properties of X-band, and negative index at $\mathrm{Ku}$-band applications. The structure of the metamaterial size is miniaturized and follows a proper EMR.

\section{Methodology}

2.1. Design of the meta-atom. The proposed meta-atom unitcell and structural parameters are illustrated in Fig. 1(a). The proposed design was developed using two C-shaped, split-circular resonators connected to each other. The thickness of all elements of the meta-atom's unit-cell is $0.035 \mathrm{~mm}$, and they are printed using copper with a conductivity of $5.8 \times 10^{7}(\mathrm{~S} / \mathrm{m})$ and with Rogers RT 6010 lossy material for the substrate material.

a)

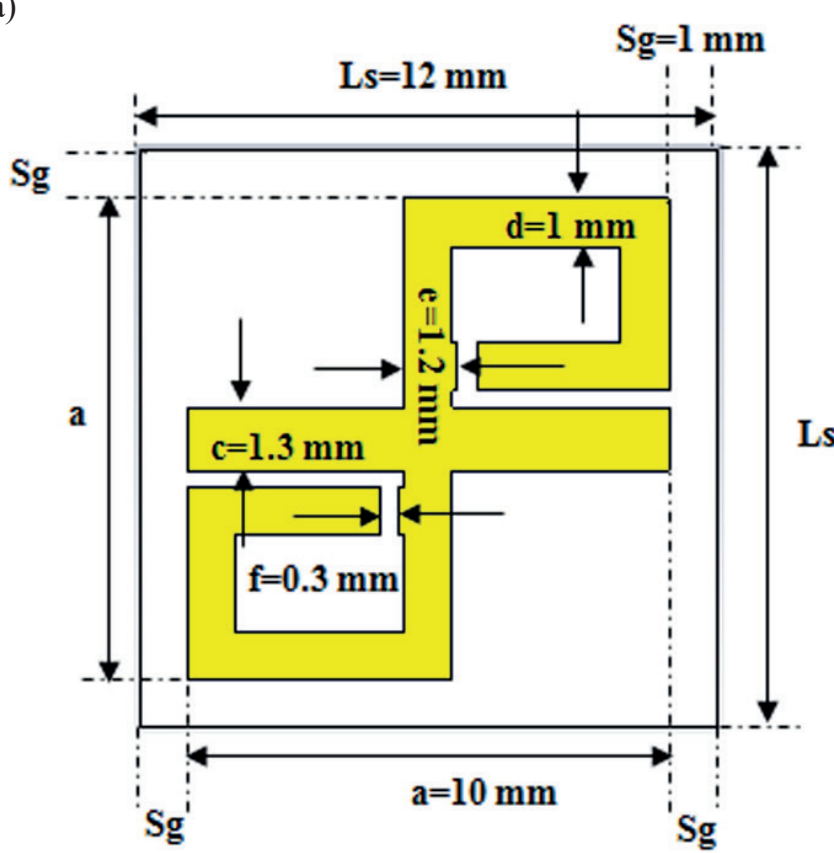

b)

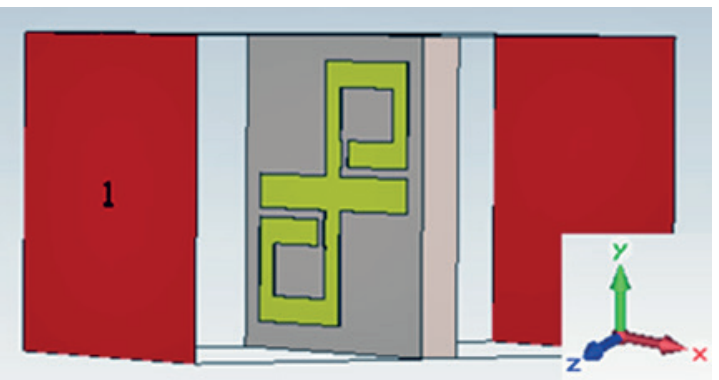

Fig. 1. a) the proposed double C-shape unit-cell structure, b) simulation setup of the proposed design

The unit-cell is fabricated on the substrate with a dielectric constant $\varepsilon, 10.2$, dielectric loss tangent $\delta, 0.0023$ and with thermal conductivity of $0.78 \mathrm{~W} / \mathrm{K} / \mathrm{m}$. The substrate of a unit-cell has the dimensions of $1.2 \mathrm{~cm}$ by $1.2 \mathrm{~cm}$, and its thickness is $0.16 \mathrm{~cm}$. In Table 1, the design parameters of the unit-cell were shown.
Table 1

Dimensions of double C-shaped unit-cell [mm]

\begin{tabular}{|l|c|c|c|c|c|c|c|}
\hline parameter & Ls & $\mathrm{a}$ & $\mathrm{c}$ & $\mathrm{d}$ & $\mathrm{e}$ & $\mathrm{f}$ & $\mathrm{Sg}$ \\
\hline value & 12 & 10 & 1.3 & 1.0 & 1.2 & 0.3 & 1.0 \\
\hline
\end{tabular}

In this paper, the CST Microwave Studio (Thomas Weiland, Germany) based on finite-difference time-domain method is adopted to investigate the design, where an incident electromagnetic wave is travelling along the positive $\mathrm{z}$-axis, with an electric field polarized along the $y$-axis. The boundary conditions of a perfect magnetic conductor and a perfect electric conductor have been utilized along the $\mathrm{x}$-axis and $\mathrm{y}$-axis respectively, and two waveguide ports have been placed along the $\mathrm{z}$-axis. A schematic diagram of a simulation of the proposed design is illustrated in Fig. 1(b). To determine the transmission coefficient and the reflection coefficient in simulation, a frequency domain solver is utilized. The impedance matching was set to fifty ohms. A frequency range $1-15 \mathrm{GHz}$ was used to simulate the design of meta-atoms.

A fabricated prototype was displayed in Fig. 2(a). The prototype consists of a $14 \times 12$ array of unit-cells made of

(a)

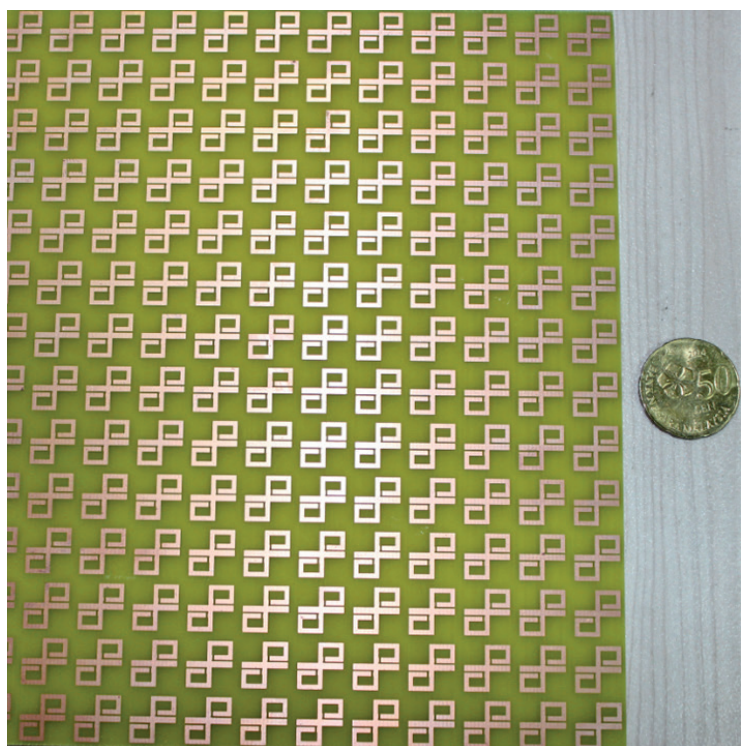

(b)

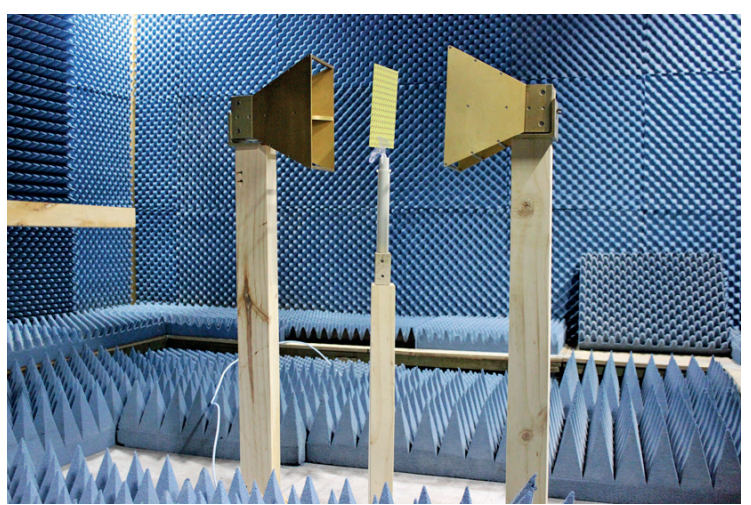

Fig. 2. a) the fabricated array of double C-shape structure, b) experimental setup 
copper materials, and the dimensions of the prototype are $168 \mathrm{~mm} \times 144 \mathrm{~mm}$. The measurement was performed [08] with the use of two horn antennas in an anechoic chamber using free-space environment.

The prototype was positioned between the horn antennas in the identical plane, similar to the simulation geometry, in order to allow the incident electromagnetic wave to travel over the prototype. The transmission co-efficient was determined with the Agilent E8363D vector network analyzer. The experimental setup of the proposed meta-atom characteristics measurement is shown in Fig. 2(b).

2.2. Scattering parameters calculations. Determining the characteristics of meta-atoms such as relative permeability and relative permittivity depends on the transmission coefficient $\left(\mathrm{S}_{21}\right)$ and reflection coefficient $\left(\mathrm{S}_{11}\right)$. A high-superiority geometrical estimation can be achieved with this design, using FDTD-method-based meshing scheme. The meshing scheme has to manage a simulation period to achieve convergent results. By using CST Microwave Studio, the S-parameters of the reported unit-cell and array were calculated. To excerpt the effective permeability $(\mu)$ and permittivity $(\varepsilon)$ from the simulated complex S-parameters, the Nicolson Rose Weir (NRW) method [17] was used. The direct refractive index method [18] was utilized to extract the effective refractive index $(\eta)$ from the simulated complex S-parameters. The simplified equation for the effective permeability, permittivity, and refractive index are as follows:

- relative effective permeability,

$$
\mu_{r}=\frac{2 c\left(1-S_{21}+S_{11}\right)}{j w d\left(1+S_{21}-S_{11}\right)},
$$

- relative effective permittivity,

$$
\varepsilon_{r}=\mu_{r}+j \frac{2 c S_{11}}{w d}
$$

- relative effective refractive index,

$$
\eta=\frac{c}{j \pi f d}\left\{\frac{\left(S_{21}-1\right)^{2}-S_{11}}{\left(S_{21}+1\right)^{2}-S_{11}}\right\}^{1 / 2} .
$$

Where, $\mathrm{S}_{11}$ and $\mathrm{S}_{21}$ are, respectively, the reflection coefficient and transmission coefficient of the meta-atom. $\mathrm{c}$ is the velocity of light, $\mathrm{d}$ is the thickness of the slab and $\mathrm{w}$ is the angular frequency.

\section{Results and discussion}

In this section, simulation and experimental results of the double $\mathrm{C}$-shaped compact meta-atom are presented. The scattering parameters and characteristics of various geometrical parameters of the design are also analyzed. The reflection coefficient $\left(\mathrm{S}_{11}\right)$ and transmission coefficient $\left(\mathrm{S}_{21}\right)$ of the unit-cell are illustrated in Fig. 3.
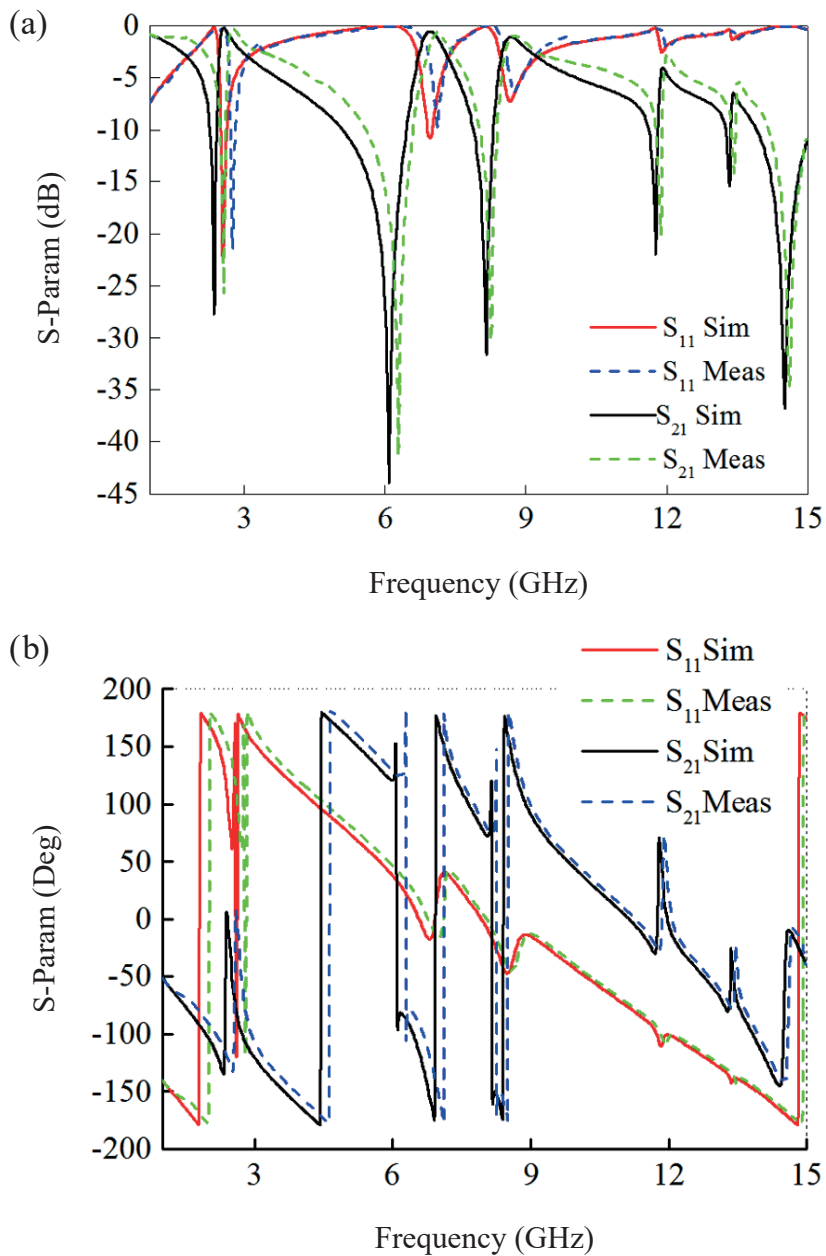

Fig. 3. Simulated and measured S-parameters response of double C-shape structure

Figure 3 demonstrates the four frequency spans of the resonance frequency, namely $2.27-2.44 \mathrm{GHz}, 5.13-6.50 \mathrm{GHz}$, and 7.9-8.34 GHz, 13.97-15.0 GHz indicating the S-band, C-band, $\mathrm{X}$-band and $\mathrm{Ku}$-band applications. The values of the transmission coefficient $\left(\mathrm{S}_{21}\right)$ at the four resonance frequencies 2.37 $\mathrm{GHz}, 6.12 \mathrm{GHz}, 8.16$, and $14.51 \mathrm{GHz}$ are $-29.32 \mathrm{~dB},-43.30$ $\mathrm{dB},-31.17 \mathrm{~dB}$, and $-36.28 \mathrm{~dB}$ respectively. From Fig. 3, it can be observed that the simulation and experimental results are in agreement with each other. Figure 4 shows the surface current distribution and the electric field of the unit-cell at 2.37 $\mathrm{GHz}, 6.12 \mathrm{GHz}, 8.16 \mathrm{GHz}$, and $14.51 \mathrm{GHz}$, where it reveals the effective negative characteristics. Moreover, in Fig. 4(a), it is shown that the distribution of surface current at the lower frequencies is higher than the highest resonance frequency of the unit-cell. According to the current distribution results, the flow of current in the upper and lower sections has the same direction at resonance frequency of $2.37 \mathrm{GHz}$. That is caused by the related magnetic fields amplifying each other, while a strong electric field is observed in Fig. 4(b).

At a resonance frequency of $6.12 \mathrm{GHz}$, the flow of current in the upper section is opposite to that in the lower section of the resonance frequency. For that reason, the related magnetic fields 


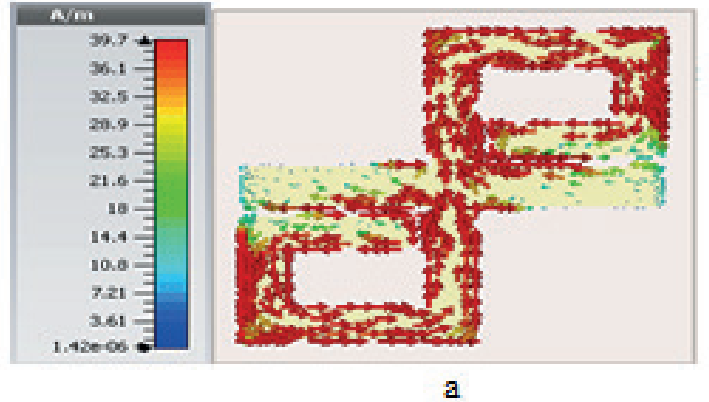

a

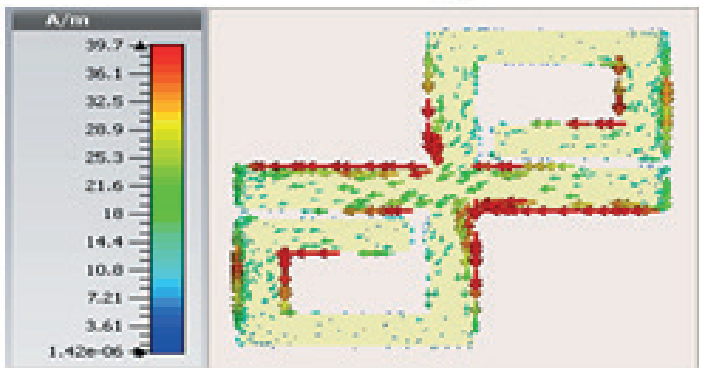

c

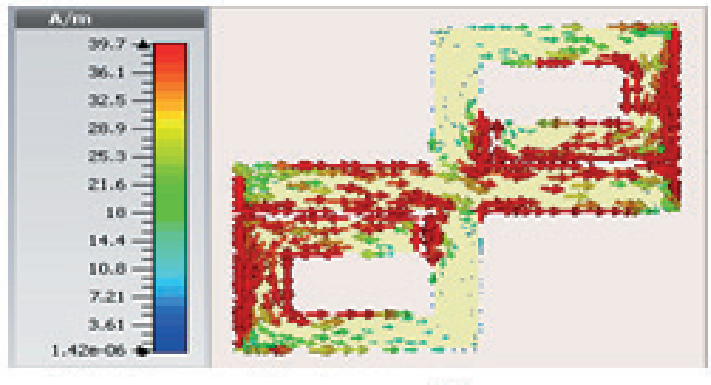

e

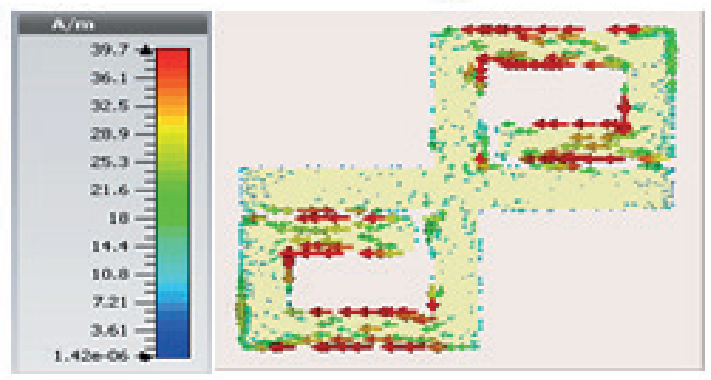

g

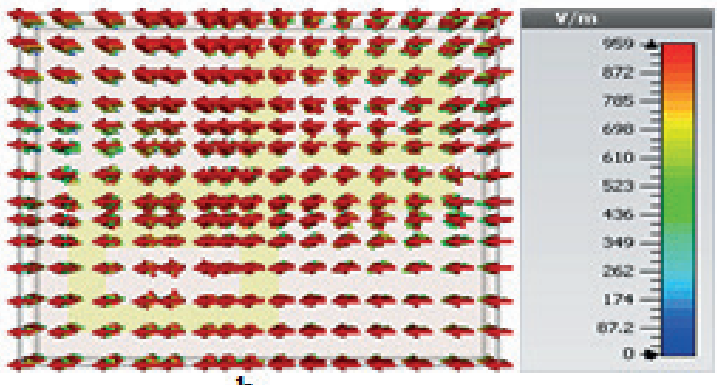

b

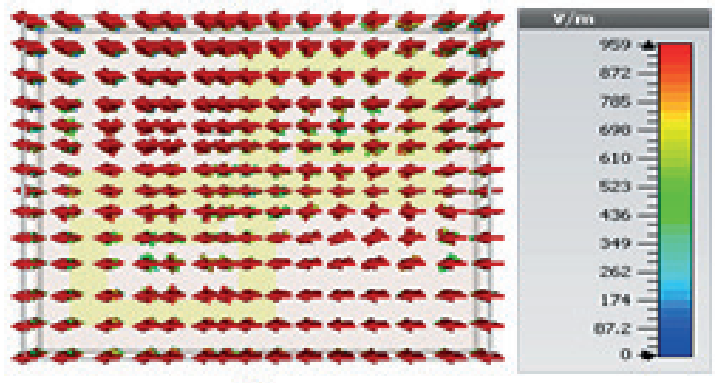

d

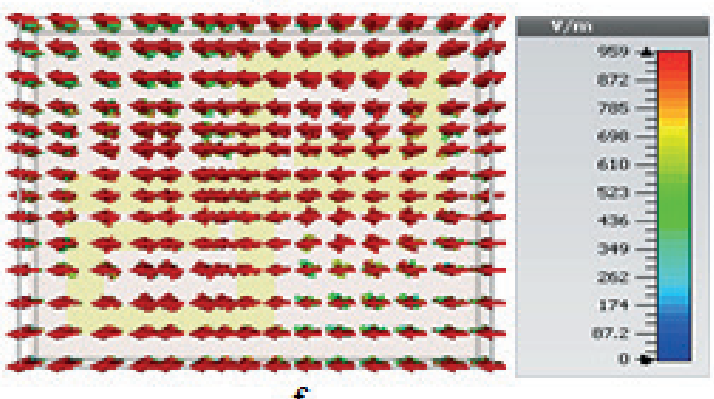

f

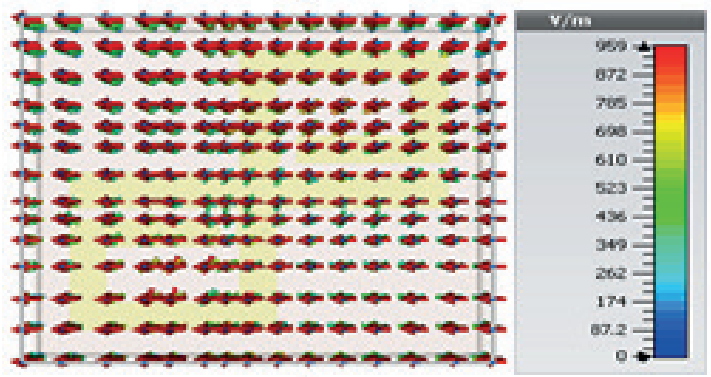

$\mathrm{h}$

Fig. 4. Current distribution and electric field at $2.37 \mathrm{GHz}, 6.12 \mathrm{GHz} 8.16 \mathrm{GHz}$ and $14.51 \mathrm{GHz}$ of double C-shape structure

nullify each other. It is also visible in Fig. 4(d), that a weak local electric field is present at the same time. In Fig. 4 (e), it can be noticed that the behavior of the current distribution is symmetrical at the resonance frequency of $8.16 \mathrm{GHz}$. For that reason, the related magnetic fields amplify each other and create a strong, local electric field, which is visible in Fig. 4(f).

Hence, it is concluded that the electric and magnetic resonances are present at these frequencies, and that the negative refractive index is noticed at frequencies shown in Fig. 6. At a resonance frequency of $14.51 \mathrm{GHz}$, the current flow of the upper section has its direction opposite to the direction of the current flow in the lower section.
This explains the related magnetic fields cancelling each other and the presence of a weak electric field, observed in Fig. 4(g, h). The frequency spans of relative permittivity $(\varepsilon)$, relative permeability $(\mu)$ and relative refractive index $(\eta)$ of the unit-cell are: $2.204-2.47 \mathrm{GHz}, 3.254-6.39 \mathrm{GHz}, 6.936-8.042$ GHz, 8.546-9.736 GHz; 6.964-14.734 GHz and 2.862-6.04 $\mathrm{GHz}, 6.6-6.908 \mathrm{GHz}, 7.356-7.972 \mathrm{GHz}, 8.112-9.694 \mathrm{GHz}$, 11.752-12.032 GHz, 13.348-13.39 GHz, 14.524-14.818 GHz, respectively. The frequency span of the double-negative region of the unit-cell is 7.356-7.972 GHz and 8.546-9.694 GHz. It can be observed, that the meta-atom has a negative refractive index at different resonance frequencies. 
(a)

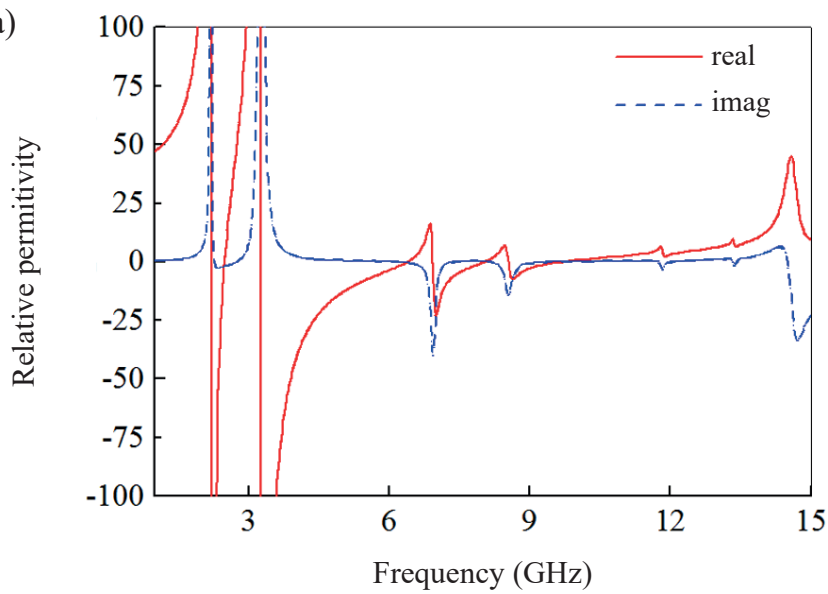

(b)

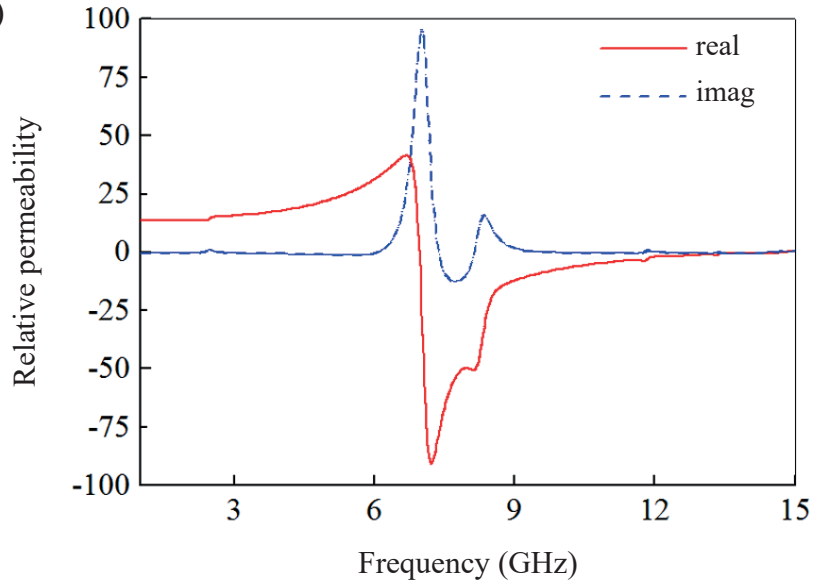

Fig. 5. Unit-cell (a) relative permittivity (b) relative permeability values of real and imaginary curves

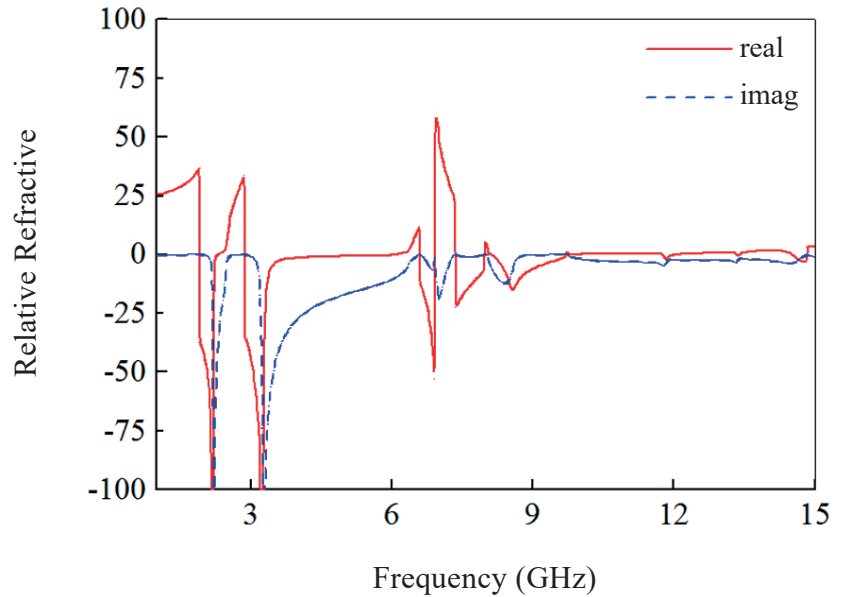

Fig. 6. Relative refractive index values of real and imaginary curves of unit-cell

Figure 5 illustrates the real and imaginary curves of the relative permittivity and relative permeability of the meta-atom. Figure 6 illustrates the relative negative refractive index curve versus frequency. The relative negative refractive indices have

(a)

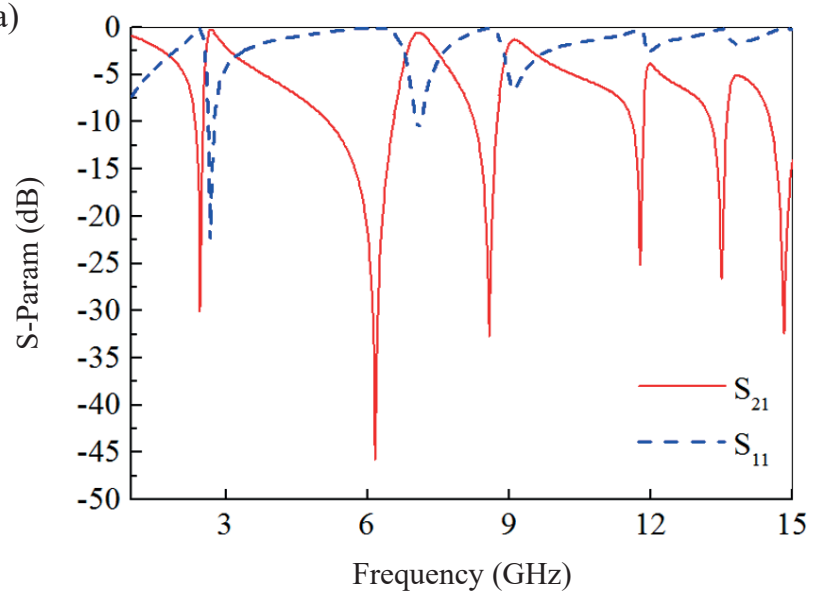

been found to be negative for the frequency spans 2.862-6.04 $\mathrm{GHz}, 6.6-6.908 \mathrm{GHz}, 7.356-7.972 \mathrm{GHz}, 8.112-9.694 \mathrm{GHz}$, 11.752-12.032 GHz, 13.348-13.39 GHz, and 14.524-14.818 GHz. The double-negative ( $\varepsilon$ and $\mu$ ) region has been found at the frequency spans 7.356-7.972 GHz and 8.546-9.694 GHz. The double-negative region proves, that the unit-cell and array of the meta-atoms act as left-handed metamaterials (LHM) that are applicable in many sophisticated areas, such as electromagnetic cloaking operation, design of filters, sensors and detectors, antenna performance enhancers, electromagnetic absorber, imaging, noise reduction, seismic protection etc.

The ratio between the wavelengths to unit-cell size, called the effective medium ratio, is important to the design of compact metamaterials. The unit-cell can reveal negative permeability and/or permittivity in the region of effective medium when the latter is greater than 4. Due to the compactness of the meta-atom, the effective medium ratio is an important matter in the field of metamaterial research. It can be seen that our proposed unit-cell size and effective medium ratio are $12 \times 12 \times 1.6 \mathrm{~mm}^{3}$ and 10.55 respectively.

3.1. Role of the changing gap of resonator (f). Figures 7-9 depict the resonator's changing gap (f) of the unit-cell from

(b)

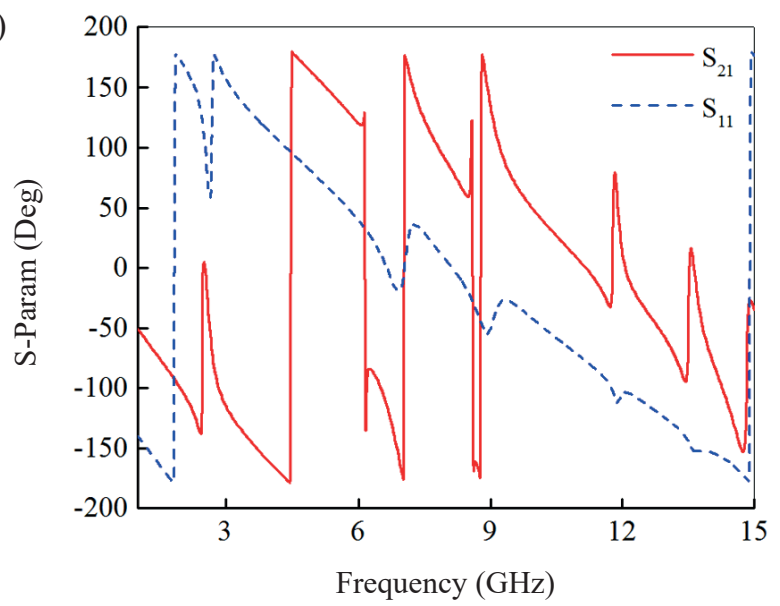

Fig. 7. S-parameters response of double C-shape structure with resonator gap width $\mathrm{c}=0.08 \mathrm{~cm}$ 
(a)

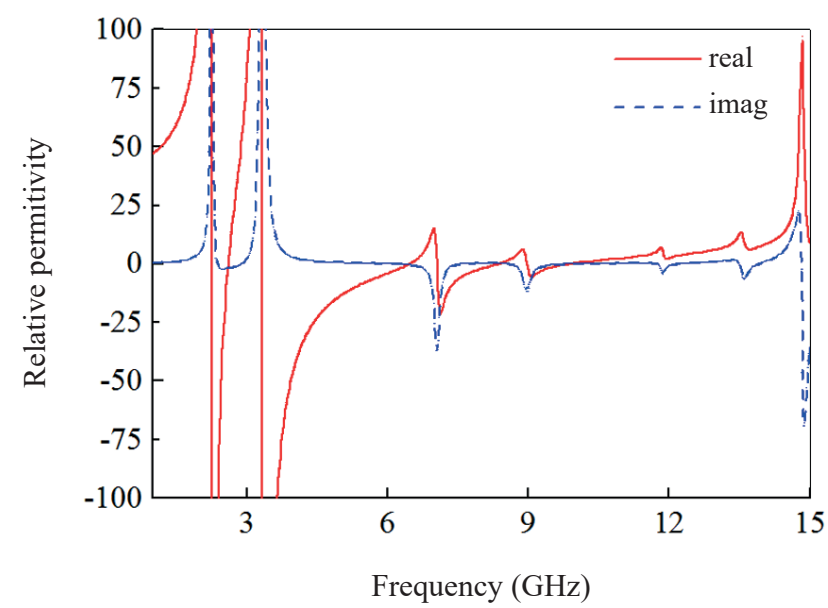

(b)

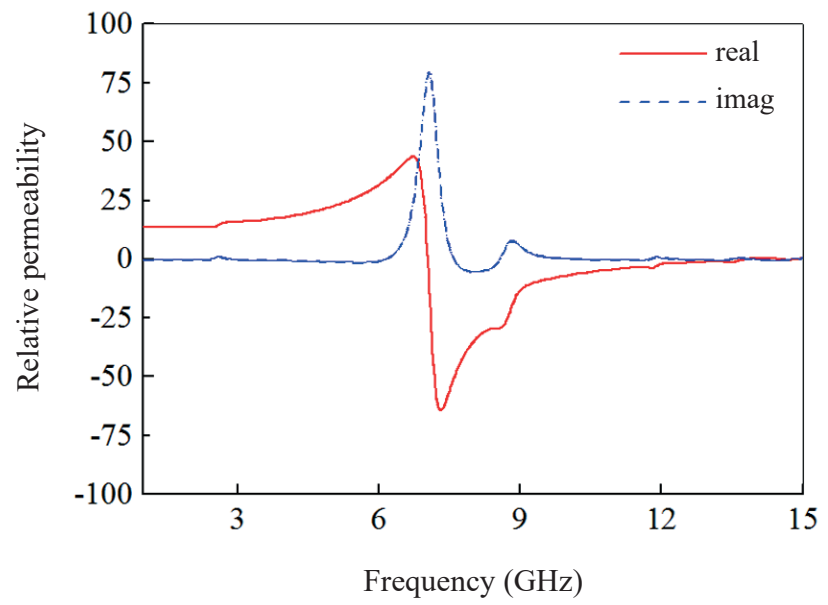

Fig. 8. Unit-cell with resonator gap width $\mathrm{c}=0.08 \mathrm{~cm}$ (a) relative permittivity, (b) relative permeability values of real and imaginary curves

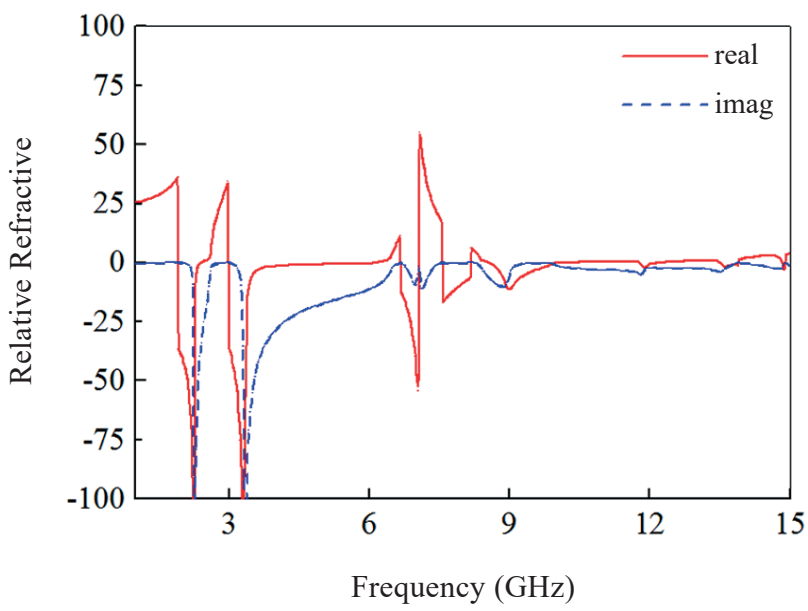

Fig. 9. Relative refractive index values of real and imaginary curves of unit-cell

Table 2

Frequency span of negative index of the unit-cell (resonator gap width $\mathrm{c}=0.08 \mathrm{~cm}$ ) of the relative effective parameters

\begin{tabular}{|l|l|}
\hline parameters & $\begin{array}{l}\text { frequency }(\mathbf{G H z}) \text { region } \\
\text { of negative index }\end{array}$ \\
\hline $\begin{array}{l}\text { relative } \\
\text { permittivity }(\boldsymbol{\varepsilon}):\end{array}$ & $\begin{array}{l}2.26-2.596,3.338-6.46,7.048-8.364, \\
8.966-9.89\end{array}$ \\
\hline $\begin{array}{l}\text { relative } \\
\text { permeability }(\boldsymbol{\mu}):\end{array}$ & $7.034-13.782,14.524-14.86$ \\
\hline $\begin{array}{l}\text { relative refractive } \\
\text { index }(\boldsymbol{\eta}):\end{array}$ & $\begin{array}{l}1.91-2.386,2.988-6.124,6.67-7.048, \\
13.502-168,8.546-10.072,11.78-12.2,\end{array}$ \\
\hline $\begin{array}{l}\text { double negative } \\
(\boldsymbol{\varepsilon} \text { and } \boldsymbol{\mu}) \text { region: }\end{array}$ & $7.048,7.566-8.168,8.966-9.89$ \\
\hline
\end{tabular}

$0.03 \mathrm{~cm}$ to $0.08 \mathrm{~cm}$ and the corresponding scattering and effective medium parameters. It is seen in Fig. 7, that the increase of the resonator gap of unit-cell leads to a right-shift of the resonance points from $2.37 \mathrm{GHz}$ to $2.47 \mathrm{GHz}, 6.12 \mathrm{GHz}$ to $6.17 \mathrm{GHz}, 8.16 \mathrm{GHz}$ to $8.60 \mathrm{GHz}$, and from $14.51 \mathrm{GHz}$ to $14.82 \mathrm{GHz}$. It is also observed that the increase in the number of resonance dips from five to six. The increase of the gap caused a reduction in the capacitance between the two ends of the resonator, and eventually, it caused a shift of the permittivity curve slightly to the right.

Table 2 depicts the details of effective negative permeability, negative permittivity, negative refractive index, and double negative region of the unit-cell.

It can be observed from Table 2 that the meta-atom with resonator gap width $\mathrm{c}=0.8 \mathrm{~cm}$ has a negative refractive index at different resonance frequencies.

3.2. Role of the resonator and middle line width ( $d$ and $c)$. The width of the square resonator (d) and middle line (c) of the unit-cell has been reduced from $0.1 \mathrm{~cm}$ to $0.05 \mathrm{~cm}$ and from $0.13 \mathrm{~cm}$ to $0.07 \mathrm{~cm}$ respectively, after that, investigating the scattering parameters and effective medium parameters that is shown in Figs. 10-12.

It can be observed from Fig. 10 that the reduction of the square resonator at the inner side and of the middle line width of the unit-cell leads to a decrease in the electric resonance at 6.12 $\mathrm{GHz}$ and $14.51 \mathrm{GHz}$ respectively. It is visible in Fig. 10 $(\mathrm{a}, \mathrm{b})$, that the resonance points were shifted slightly to the left side in relation to the base of the unit-cell. This is due to an increase in the capacitance between the resonator and the midline. Similarly, an incrementation of the width of the resonator at the outer side would shift the transmittance to right side.

Figure 11demonstrates the real and imaginary curves of the relative permittivity and relative permeability of the unitcell, with resonator and middle line width changed simultaneously. It can be seen in Fig. 11, that the magnitude of 
(a)

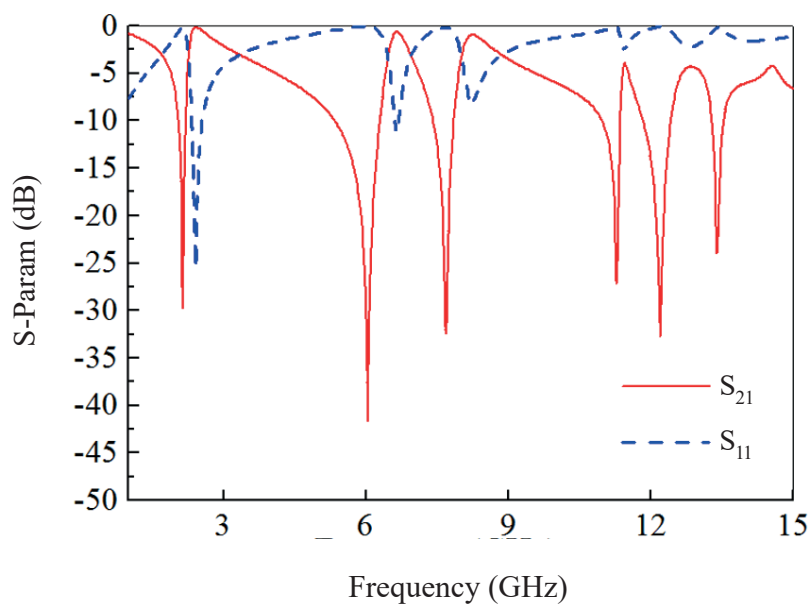

(b)

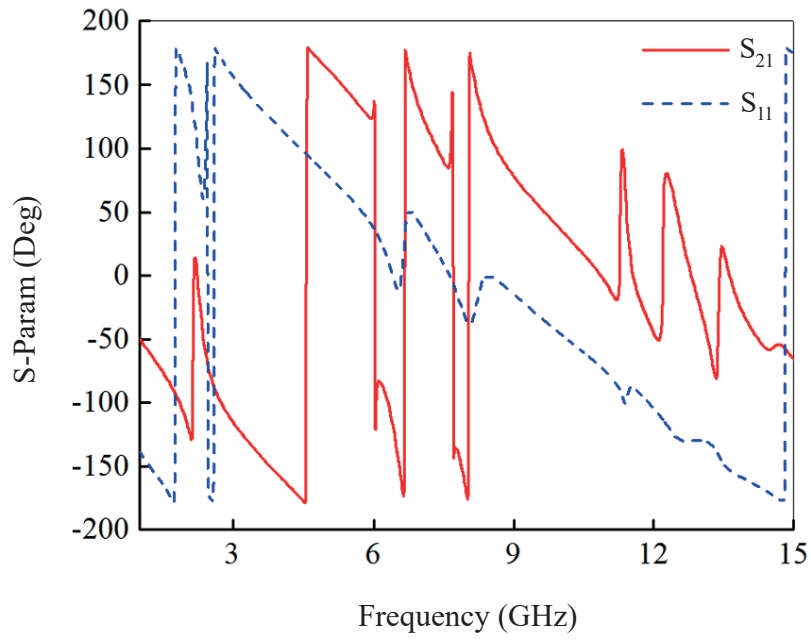

Fig. 10. S-parameters response of double C-shape structure with reduced resonator and middle line width (d and c)

(a)

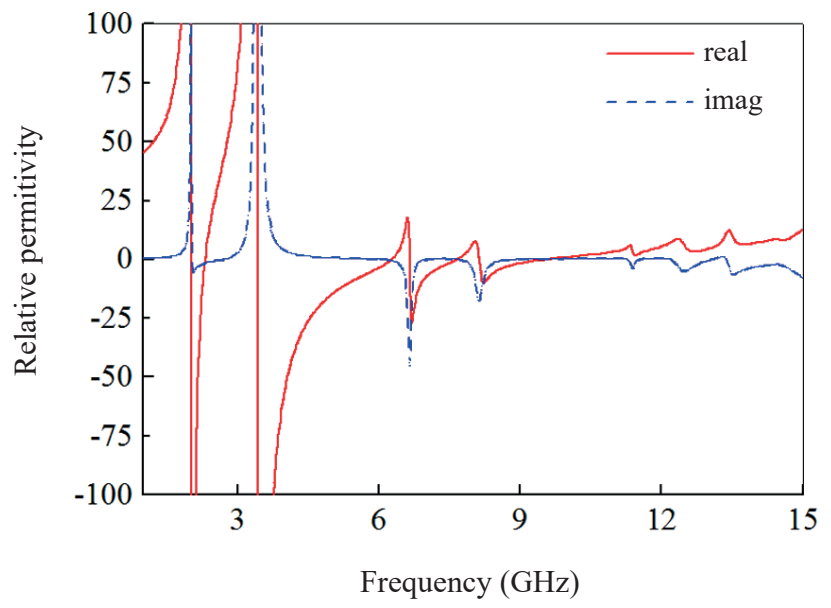

(b)

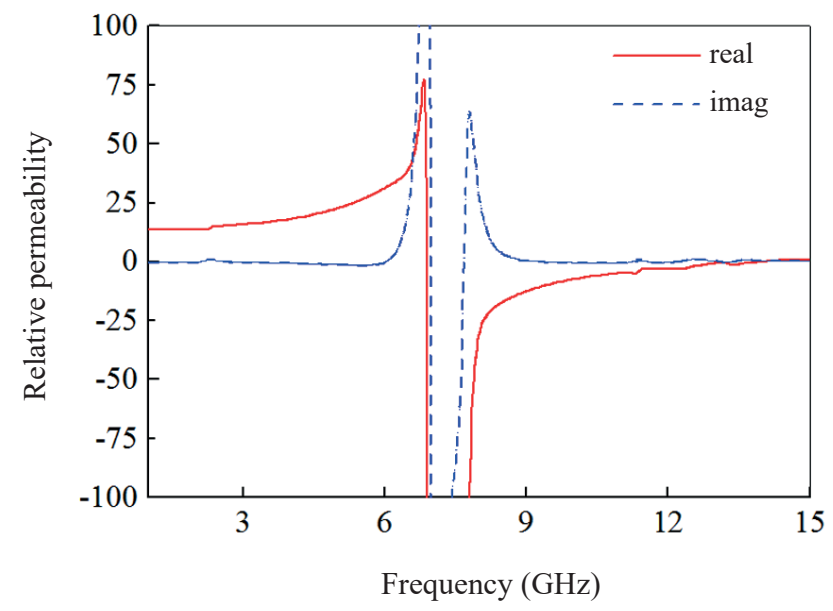

Fig. 11. Unit-cell of reduced resonator and middle line width (d and c). (a) relative permittivity, (b) relative permeability values of real and imaginary curves

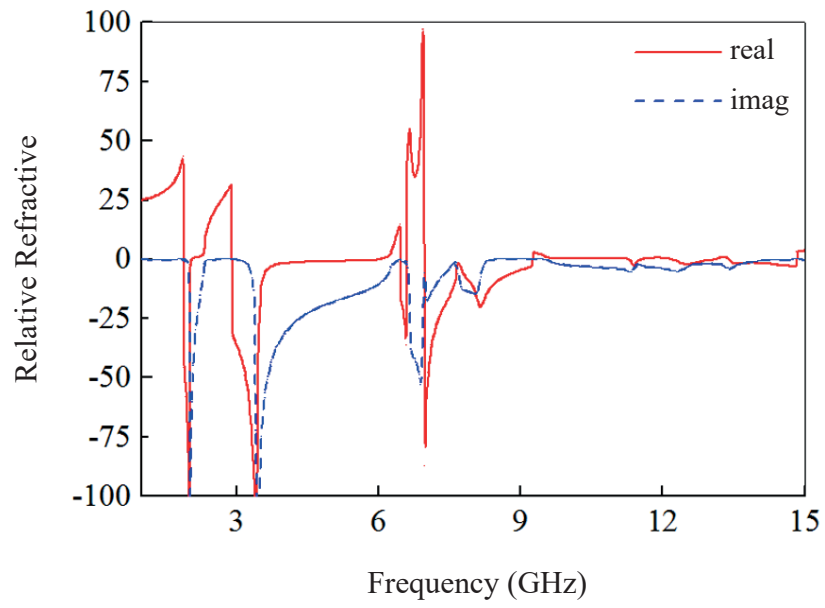

Fig. 12. Relative refractive index values of real and imaginary curves of unit-cell

the real and imaginary curves of the relative permittivity has slightly shifted to left and that the amplitude of the real part has reached a negative frequency of nearly $3 \mathrm{GHz}$. The relative permeability of this unit-cell was found to be constant. On the other hand, the magnitude of the permittivity curves is stronger than the basis of unit-cell. Figure 12 also illustrates the relative negative refractive index curve versus the frequency of the unit-cell. The relative negative refractive index originated to be negative for a frequency span 4.598-10.268 $\mathrm{GHz}$ and $10.618-13.236 \mathrm{GHz}$. The double negative $(\varepsilon$ and $\mu)$ region has been found at the frequency spans 9.176-10.142 $\mathrm{GHz}$ and $11.458-12.34 \mathrm{GHz}$.

The magnitude of the relative refractive index curves is weaker than the basis of the unit-cell, because of the reduction of the inductive path. The effective negative values of the relative permittivity, relative permeability, and relative refractive index of unit-cell with reduced resonator and middle line width 
metamaterial, can be observed in Table 3 . The double negative properties, visible in Table 3 , are what makes the meta-atoms left-handed metamaterials.

Table 3

Frequency span of negative index of the unit-cell (reduced resonator and middle line width) of the relative effective parameters

\begin{tabular}{|l|l|}
\hline parameters & $\begin{array}{l}\text { frequency }(\mathbf{G H z}) \text { region } \\
\text { of negative index }\end{array}$ \\
\hline $\begin{array}{l}\text { relative permittivity } \\
(\boldsymbol{\varepsilon}):\end{array}$ & $\begin{array}{l}2.834-3.198,4.668-8.266, \\
9.176-10.142,11.458-12.34\end{array}$ \\
\hline $\begin{array}{l}\text { relative permeability } \\
(\boldsymbol{\mu}):\end{array}$ & $7.23-13.936$ \\
\hline $\begin{array}{l}\text { relative refractive } \\
\text { index }(\boldsymbol{\eta}):\end{array}$ & $4.598-10.268,10.618-13.236$, \\
\hline $\begin{array}{l}\text { double negative } \\
(\boldsymbol{\varepsilon} \text { and } \boldsymbol{\mu}) \text { region: }\end{array}$ & $9.176-10.142,11.458-12.34$ \\
\hline
\end{tabular}

Table 3 depicts the details of effective negative permeability, negative permittivity, negative refractive index, and double negative region of the unit-cell. It can be observed in Table 3, that the meta-atom with the reduced resonator and middle line width has a negative refractive index at different resonance frequencies. Due to the compactness of the meta-atom, the effective medium ratio is an important matter in the field of metamaterial research. A comparison of the proposed meta-atom and other meta-atoms (metamaterials) has been performed and shown in Table 4 . The parameters of the

Table 4

Comparison of resonance number, covered band, and EMR with other unit-cells

\begin{tabular}{|l|c|l|c|}
\hline \multicolumn{1}{|c|}{ ref. No. } & $\begin{array}{c}\text { No. of } \\
\text { resonance } \\
\text { in S21 }\end{array}$ & \multicolumn{1}{|c|}{$\begin{array}{c}\text { covered } \\
\text { band }\end{array}$} & $\begin{array}{c}\text { effective } \\
\text { medium } \\
\text { ratio }\end{array}$ \\
\hline $\begin{array}{l}\text { Fabio Urbani } \\
\text { et al. } 2010[08]\end{array}$ & 1 & X-Band & 1.2 \\
\hline $\begin{array}{l}\text { Anik Mallik } \\
\text { et al. 2013 [10] }\end{array}$ & 1 & C-Band & 1.99 \\
\hline $\begin{array}{l}\text { Theodosios } \\
\text { et al. 2012 [11] }\end{array}$ & 1 & C-Band & 6.9 \\
\hline $\begin{array}{l}\text { Sikder } \\
\text { et al. 2015 [12] }\end{array}$ & 5 & $\begin{array}{l}\text { S, X- and } \\
\mathrm{K}_{\mathrm{u}} \text {-Band }\end{array}$ & 4.32 \\
\hline $\begin{array}{l}\text { Behnam } \\
\text { et al. } 2015[13]\end{array}$ & 2 & S-Band & 12.5 \\
\hline $\begin{array}{l}\text { Abhishek } \\
\text { et al. 2015 [14] }\end{array}$ & 1 & C-Band & 7.14 \\
\hline $\begin{array}{l}\text { Proposed } \\
\text { Meta-atom }\end{array}$ & 5 & $\begin{array}{l}\text { S, C, X- and } \\
\mathrm{K}_{\mathrm{u}} \text {-Band }\end{array}$ & 10.55 \\
\hline
\end{tabular}

meta-atom have been considered as tools for performance verification, namely number of resonance frequencies, covered band, and effective medium ratio etc. The proposed meta-atom cannot maintain higher effective medium ratio as compared to [13], although more resonance numbers with more covered band numbers have also been achieved. Hence, the proposed meta-atom can attain better compactness while maintaining a more widely covered band and a higher count of resonance dips than the designs in $[8,10,11,13,14]$. The proposed meta-atom showed five resonances, a higher effective medium ratio and multiband such as $\mathrm{S}-, \mathrm{C}-, \mathrm{X}-$ and $\mathrm{K}_{\mathrm{u}}$-Band, which are suitable for sophisticated microwave applications.

\section{Conclusions}

A new effective medium ratio obeying compact meta-atom structure of the unit-cell for multiband applications was studied in this paper. The scattering constitutive parameters and the effective parameters of the proposed meta-atom were analyzed over the frequency span. The results show negative-index properties of the unit-cell structure in a multiband frequency range. Furthermore, the unit-cell structure of the proposed meta-atom is reduced in size, covers more bands, and follows a better effective medium ratio with a value of 10.55 , which is more suitable in microwave spectra. The free-space measurement method was used to verify the results of a prototype of the meta-atom that was fabricated and tested. The parametric studies were conducted, and the meta-atom acted as a left-handed metamaterial. This meta-atom can prove suitable for many applications, for example for energy absorbers, electromagnetic cloaking operations, electromagnetic energy harvesters, microwave sensors and detectors, antenna performance enhances, high data rate communications etc.

\section{REFERENCES}

[1] J. B. Pendry, A. J. Holden, D. J. Robbins, and W. J. Stewart, "Magnetism from conductors and enhanced nonlinear phenomena", IEEE Trans Microw. Theory Tech. 47 (11), 2075-2084 (1999).

[2] R. A. Shelby, D. R. Smith, and S. Schultz, "Experimental verification of a negative index of refraction", Sci. 292, 77-79 (2001).

[3] S. S. Islam, M. R. I. Faruque, M. J. Hossain, and M. T. Islam, "Limitations of metamaterials for invisibility cloaking", J.Tek. 78, 1-5 (2016).

[4] W. Withayachumnankul, C. Fumeaux, and D. Abbott, "Compact electric-LC resonators for metamaterials", Opt.. Exp. 18, 25912-25921 (2010).

[5] D. Schurig, J. J. Mock, and D. R. Smith, "Electric-field-coupled resonators for negative permittivity metamaterials", Appl. Phys. Lett. 88, 1-3 (2006).

[6] J. Zhou, L. G. Tuttle, T. Koschny, and C. M. Soukoulis, "Negative index materials using simple short wire pairs", Phys. Rev. B 73, 1-4 (2006).

[7] A. Dhouibi, S. N. Burokur, A. Lustrac, A. Priou, "Study and analysis of an electric Z-shaped meta-atom", Adv. Electrom. 1, 64-70 (2012). 
[8] F. Urbani, "Experimental analysis of novel single-sided lefthanded metamaterial", IEEE Antennas and Wirel. Propag. Lett. 9, 720-723 (2010).

[9] N. Naeem, A. Ismail, A. R. H. Alhawari, M. A. Mahdi, "Terahertz dielectric sensor based on novel hexagon meta-atom cluster", Appl. Comput. Electromagn.Soc.J. 30, 996-1002 (2015).

[10] A. Mallik, A. S. Kundu, M. O. Goni, "Design of a novel two-rectangular U-shaped double negative metamaterial", In Proceedings of the Inter. Conf. Info. Electronics \& Vision (ICIEV), Dhaka, Bangladesh, 17-18 (2013).

[11] T. D. Karamanos, A. I. Dimitriadis, and N. V. Kantartzis, "Compact double-negative metamaterials based on electric and magnetic resonators", IEEE Antennas and Wirel. Propag. Lett. 11, 480-483 (2012)

[12] S. S. Islam, M. R. I. Faruque, and M. T. Islam, "Design and absorption analysis of a new multi-band split-S-shaped metamaterial", Sci.Eng. Compos. Mater. DOI 10.1515/ secm2014-0376:1-10 (2015).
[13] B. Zarghooni, A. Dadgarpour, T. A. Denidni, "Greek-key pattern as a miniaturized multiband metamaterial unit-cell", IEEE Antennas and Wirel. Propag. Lett. 14, 1254-1257 (2015).

[14] A. Sarkhel, D. Mitra, S. Paul, S. R. B. Chaudhury, "A compact meta atom for dual band negative permittivity metamaterial", Microw. Opt. Tech. lett. 57 (5), 1152-1156 (2015).

[15] A. Sarkhel, D. Mitra, S. Paul, S. R. B. Chaudhury, "A compact metamaterial with multi-band negative-index characteristics", Appl. Phys. A 122, 1-10 (2016).

[16] C. Sabah, T. Nesimoglu, "Design and characterization of a resonator-based metamaterial and its sensor application using microstrip technology", Opt. Eng. 55(2), 1-7 (2016).

[17] A. M. Nicolson, G. F. Ross, "Measurement of the intrinsic properties of materials by time-domain techniques", IEEE Trans. Instrument. Meas. 19, 377-382 (1970).

[18] S. S. Islam, M. R. I. Faruque, and M. T. Islam, "A new direct retrieval method of refractive index for the metamaterial", Current Sci. 109, 337-342 (2015). 